\title{
ATUAÇÃO MULTIDISCIPLINAR NO TRATAMENTO DA EPIDERMÓLISE BOLHOSA ${ }^{1}$
}

\author{
MULTIDISCIPLINARY ACTION IN THE TREATMENT OF \\ EPIDERMOLYSIS BULLOSA
}

\author{
Gustavo Coringa de Lemos $^{2}$ \\ Ivanna Trícia Gonçalves Fernandes ${ }^{3}$ \\ Pâmela Reis Vidal ${ }^{4}$
}

RESUMO: OBJETIVO: evidenciar a atuação multidisciplinar no tratamento dos indivíduos acometidos pela EB. MÉTODOS: trata-se de uma revisão de literatura, onde artigos científicos sobre a temática foram pesquisados nas bases de dados: SCIELO (Scientific Eletronic Library Online), BVS (Biblioteca Virtual em Saúde) e PubMED, publicados nos últimos 15 anos (2006-2021), aplicando-se os seguintes descritores: Epidermólise Bolhosa e Multidisciplinar. RESULTADOS: os resultados mostram que a abordagem sobre a atuação multidisciplinar no manejo da patologia ainda é citada de forma discreta nos estudos científicos, pois apesar desta atuação ser considerada importante para proporcionar aos indivíduos um tratamento ainda mais efetivo, são poucas as relações expostas nos estudos entre os profissionais de saúde que podem contribuir para o tratamento do indivíduo acometido e dos seus familiares. CONCLUSÃO: através do presente estudo pôde-se evidenciar que a literatura apresenta uma escassez de estudos científicos sobre esta temática, principalmente sobre a atuação multiprofissional nos cuidados destes pacientes e seus familiares. Diante disso, surge a necessidade de realização de mais estudos sobre a EB, especialmente enfatizando a atuação multidisciplinar no manejo da patologia, para que assim estes estudos possam apoiar a prática dos profissionais de saúde.

PALAVRAS CHAVE: Epidermólise Bolhosa. Equipe de Assistência ao Paciente. Doença Crônica.

\footnotetext{
${ }^{1}$ O presente trabalho foi realizado com apoio da Coordenação de Aperfeiçoamento de Pessoal de NívelSuperior - Brasil (CAPES) - Código de Financiamento 001.

${ }^{2}$ Fisioterapeuta. Mestrando em Cognição, Tecnologias e Instituições pela Universidade Federal Ruraldo Semi-Árido (UFERSA).gustavocoringafisio@gmail.com.

${ }^{3}$ Fisioterapeuta. Mestre em Neuroengenharia pelo Instituto Internacional de Neurociências Edmond eLily Safra (IIN-ELS). ivannatricia@gmail.com.

${ }^{4}$ Fisioterapeuta. Mestranda em Ciências da Atividade Física pela Universidade de São Paulo (USP).pamelareisvidal@gmail.com.
} 
ABSTRACT: OBJECTIVE: evidence the multidisciplinary action in the treatment of individuals affected by EB. METHODS: it is a literature review, where scientific articles on the subject were searched in the databases: SCIELO (Scientific Electronic Library Online), VHL (Virtual Health Library) and PubMED, published in the last 15 years (2006-2021), applying the following descriptors: Epidermolysis Bullosa and Multidisciplinary. RESULTS: the results show that the approach on multidisciplinary performance in the management of pathology is still cited in a way discreet in scientific studies, because despite this acting be considered important to provide individuals with an even more effective treatment, few are relationships exposed in the studies among health professionals who can contribute for the treatment of affected individual and their family members. CONCLUSION: through the present study it was evident that the literature presents a scarcity of scientific studies on this theme, mainly about the multiprofessional performance in the care of these patients and their families. That said, there is a need for realization more studies on EB, especially emphasizing the multidisciplinary performance in the management of pathology, so that these studies can support the practice of health professionals.

KEYWORDS: Epidermolysis Bullosa. Patient Care Team. Chronic Disease. 


\section{INTRODUÇÃO}

A Epidermólise Bolhosa (EB) envolve um grupo heterogêneo de doenças, hereditárias ou adquiridas, relativamente raras, que possui como característica o desenvolvimento de bolhas na região cutâneo-mucosa. As bolhas podem surgir após traumatismos ou irritações, assim como podem ocorrer de forma espontânea, podendo ou não deixar cicatrizes na epiderme (SILVA et al., 2011).

A EB pode manifestar-se em qualquer fase da vida (AMARAL, ANDRADE \& BARBOSA, 2014). Possuem três tipos principais, os quais são classificados de acordo com o nível histológico de formação da bolha: simples, juncional e distrófica, além da inclusão de um quarto grupo (Síndrome de Kindler), após o terceiro consenso de EB (OLIVEIRA, 2010; BOEIRA, 2012).

A EB caracteriza-se por produzir vesículas na pele (como mãos e pés) e, em alguns casos, nas membranas mucosas. As fibras proteicas de colágeno, que ligam as camadas da pele, não funcionam eficazmente, assim separando-se facilmente. $O$ espaço que se forma entre elas é preenchido por soro ou fluido rico em proteínas, dessa forma dando origem a bolha (AMARAL, ANDRADE \& BARBOSA, 2014).

$O$ indivíduo acometido pela EB pode apresentar bolhas nas superfícies externas e internas do corpo, atrofia da pele no dorso das mãos, com perda parcial de impressões digitaise hiperqueratose nas mãos e nos pés. A evolução se dá com o aparecimento de vários ciclos de formação de bolhas, geralmente com infecção secundária (ALVES et al., 2007).

Em alguns casos de EB, as bolhas variam entre superficiais e até mais profundas, ocasionando no aumento do seu tamanho, podendo estas ser dolorosas e com sangue, tendo em vista que a separação das camadas se localizam abaixo da junção da epiderme com a derme, onde se encontram vasos sanguíneos e nervos. Além disso pode ocorrer também caimento das unhas e feridas que podem evoluir para infecções ou deixar cicatrizes que levam a complicaçõescomo pseudosindactilia e distrofia das mãos, que ocasionam na perda dos movimentos dos dedos, dessa 
forma dificultando a socialização do indivíduo acometido com o meio em que vive (BEGA et al., 2015).

Além da pele e do sistema musculoesquelético, outros órgãos também podem ser afetados nos indivíduos acometidos pela EB, especialmente aqueles com forma recessiva da patologia. Além de bolhas, outras doenças de pele também podem ser observadas nestes indivíduos, tais como: neoplasma cutâneo, eczema, dermatite atópica e alergias. Dentre os órgãos que são mais afetados nos indivíduos com EB, destacam-se: os dentes, o trato gastrintestinal, o trato respiratório superior, o trato geniturinário, os olhos e o sistema cardiovascular (DAS \& SAHOO, 2004; BARROS, RASKIN \& PEREIRA, 2004).

O diagnóstico da EB é clínico e laboratorial, mas é de suma importância considerar a história familiar e a consanguinidade dos pais (ALVES et al., 2007). A confirmação exata da doença pode ser realizada através da imunofluorescência, do mapeamento genético e antígenos monoclonais específicos, além da microscopia eletrônica, sendo esta considerada padrão-ouro no diagnóstico da EB (BEGA et al., 2015).

De acordo com o estudo de AMARAL, ANDRADE \& BARBOSA (2014), A EB é uma afecção crônica e possui variados graus de intensidade, o que requer uma abordagem interdisciplinar para proporcionar aos indivíduos acometidos e os seus familiares uma assistência eficaz, bem como a compreensão da doença e dos cuidados necessários, para que estes sejam mantidos no domicílio. Além disso, o estudo evidencia que o indivíduo acometido por EB requer intervenções visando à prevenção do aparecimento de sequelas, o que pode dificultar a sua integração social.

A EB torna o indivíduo acometido extremamente frágil, e, devido a isso, necessitam de cuidados que possuam como objetivo evitar novos traumas e ulcerações. Sendo assim, o planejamento assistencial feito a partir da avaliação da equipe multidisciplinar deve garantir que o indivíduo tenha um cuidado adequado à sua pele e mucosas, evitando mais e maiores complicações no sentido de desencadear ou agravar o surgimento de lesões (AMARAL, ANDRADE \& BARBOSA, 2014). 
Diante disso, o presente estudo tem como objetivo evidenciar a atuação multidisciplinarno tratamento dos indivíduos acometidos pela EB. O estudo justifica-se pelo fato da EB se tratar de uma doença crônica, que possui diferentes graus de intensidade e acometimentos, requerendo para o seu manejo uma abordagem multiprofissional, tendo em vista que, além dos indivíduos acometidos, os familiares destes também precisam de cuidados e/ou orientações para o manejoadequado da patologia.

\section{METODOLOGIA}

O presente estudo trata-se de uma revisão de literatura, que de acordo com Tomasi e Yamamoto (1999, p.3) "é uma sistemática de leituras e registros que acontecem ao longo do processo de pesquisa". Diante disso, o desenvolvimento deste estudo foi divido em 4 etapas, assim distribuídas:

\section{$1^{\text {a }}$ Etapa - Fontes}

Nesta etapa foi realizado o levantamento bibliográfico, que de acordo com CERVO \& BERVIAN (2002), tem como finalidade levantar todas as referências encontradas sobre um determinado tema. Assim, artigos científicos sobre a temática do presente trabalho foram pesquisados nas bases de dados: SCIELO (Scientific Eletronic Library Online), BVS (Biblioteca Virtual em Saúde) e PubMED, publicados nos últimos 15 anos (2006-2021), sendoeste período considerado devido à escassez de publicações sobre a temática. Para a realização da pesquisa, os seguintes descritores foram aplicados: Epidermólise Bolhosa e Multidisciplinar. As pesquisas foram realizadas nas bases de dados no período de fevereiro de 2021.

Foram utilizados artigos disponíveis online com acesso livre (gratuito), escritos nas línguas Inglesa e Portuguesa, e em texto completo. Para a seleção das fontes 
foram usados como critérios de inclusão: artigos completos que abordassem a temática proposta, e como critério de exclusão: artigos que não estivessem nas Línguas Inglesa e/ou Portuguesa.

\section{$2^{\mathrm{a}}$ Etapa - Coleta de dados}

$\mathrm{Na}$ presente etapa foi realizada a leitura exploratória de todo o material selecionado, objetivando analisar se os artigos em questão respondem aos questionamentos do presente trabalho. Em seguida foi realizada a leitura seletiva destes, aprofundando nas partes de maioresinteresses. Foi realizado ainda o registro das informações extraídas dos artigos em instrumentoespecífico (título, autores, ano, base de dados e resultados).

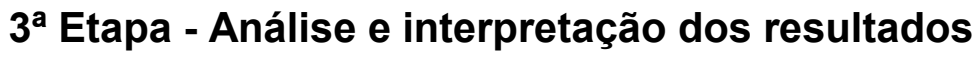

Nesta etapa, foram realizadas leituras analíticas para ordenar as informações das fontes, para que se desenvolvesse a pesquisa. Na tabela 1, pode ser observado o número de artigos encontrados em cada etapa da presente coleta de dados. 
Tabela 1 - Seleção dos artigos científicos.

\begin{tabular}{|l|l|}
\hline \multicolumn{1}{|c|}{ ATIVIDADE } & \multicolumn{1}{c|}{ NÚMERO DE DOCUMENTOS } \\
\hline Busca nas bases de dados & 14 artigos (SCIELO 2; BVS 9; PubMED 3) \\
\hline Exclusão por ano (2006 - 2021) & 14 artigos (SCIELO 2; BVS 9; PubMED 3) \\
\hline Texto completo & 13 artigos (SCIELO 2; BVS 8; PubMED 3) \\
\hline $\begin{array}{l}\text { Seleção de Artigos nas Línguas } \\
\text { Portuguesa e Inglesa }\end{array}$ & 8artigos (SCIELO 1; BVS 4; PubMED 3) \\
\hline Seleção de artigos com livre acesso & 4 artigos (SCIELO 1; BVS 2, PubMED 1) \\
\hline Exclusão de artigos repetidos & 3 artigos (SCIELO 1; BVS 1, PubMED 1) \\
\hline Artigos finais & 3 artigos \\
\hline
\end{tabular}

Autoria própria (2021).

\section{$4^{\mathrm{a}}$ Etapa - Discussão dos resultados}

As categorias encontradas na etapa citada anteriormente foram discutidas a partir do referencial teórico sobre a temática do assunto. A partir dos dados coletados, serão apresentadase, consequentemente, realizadas as discussões desse trabalho.

Para melhor exposição dos artigos científicos selecionados no presente estudo, de acordo com as características citadas anteriormente, foi elaborada a tabela 2, composta pelos seguintes dados de cada artigo: "título", "autores", "ano", "bases de dados" e "resultados".

\section{RESULTADOS E DISCUSSÃO}

Apesar da complexidade que envolve a EB, para BARBOSA et al. (2005) ainda não háum tratamento curativo da $\mathrm{EB}$, sendo assim o suporte clínico objetiva a prevenção e tratamento de novas bolhas, das infecções, retrações e sinéquias. Para o autor, a melhora da qualidade de vida dos indivíduos acometidos deve estar entre os objetivos da equipe multiprofissional e dosfamiliares. 
Diante disso, o presente estudo buscou evidenciar, através de publicações científicas já existentes na literatura, como é realizada a atuação multidisciplinar na atenção ao indivíduo acometido pela EB, bem como o suporte aos familiares destes. Dessa forma, chegou-se aos resultados apresentados na tabela 2.

Tabela 2 - Resultados dos estudos explorados.

\begin{tabular}{|c|c|c|c|c|}
\hline TíTULO & AUTORES & ANO & $\begin{array}{l}\text { BASES DE } \\
\text { DADOS }\end{array}$ & RESULTADOS \\
\hline $\begin{array}{l}\text { Atendimento } \\
\text { multidisciplinar } \\
\text { do paciente } \\
\text { do } \\
\text { ortodôntico com } \\
\text { Epidermólise } \\
\text { Bolhosa }\end{array}$ & $\begin{array}{l}\text { ALVES et al., } \\
2007 .\end{array}$ & 2007 & Scielo & 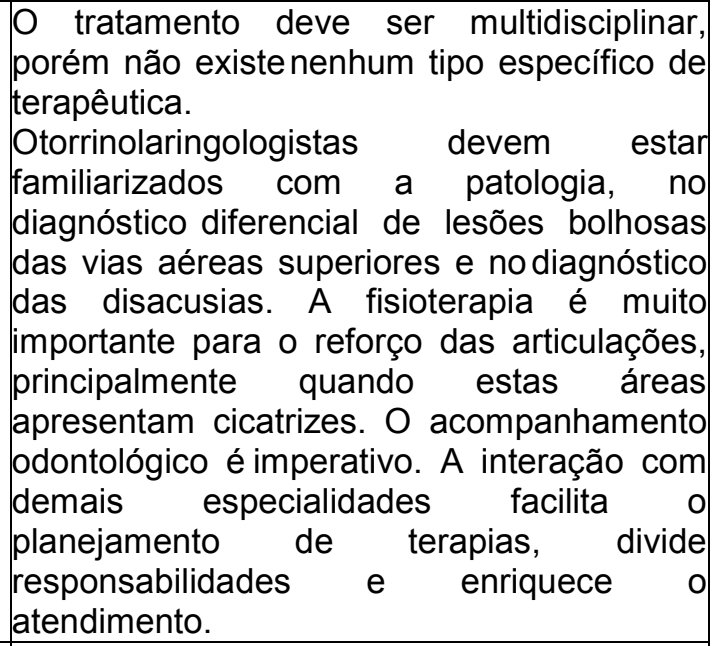 \\
\hline $\begin{array}{l}\text { The challenges } \\
\text { of meeting } \\
\text { nutritional } \\
\text { requirements in } \\
\text { children and } \\
\text { adults with } \\
\text { epidermolysis } \\
\text { bullosa: } \\
\text { proceedings of a } \\
\text { Multidisciplinary } \\
\text { team study day }\end{array}$ & $\begin{array}{l}\text { HUBBARD et } \\
\text { al., } 2011 .\end{array}$ & 2011 & BVS & $\begin{array}{l}\text { Não há diretrizes claras sobre quando tratar } \\
\text { a anemia, nem o melhor método para fazer } \\
\text { isso. } \\
\text { Evidencia-se a necessidade de se realizar } \\
\text { uma investigação mais aprofundada sobre } \\
\text { os diversos fatores que influenciam o } \\
\text { fornecimento de melhores práticas em } \\
\text { suporte nutricional para pessoas com EB. A } \\
\text { nutrição ideal é a base do manejo na } \\
\text { promoção da melhor qualidade de vida } \\
\text { possível para os pacientes e suas famílias. } \\
\text { Paraisso, os profissionais devem trabalhar } \\
\text { em conjunto e disponibilizar orientações } \\
\text { consistentes e imparciais. Ressaltou-se a } \\
\text { importância de otimizar o crescimento e a } \\
\text { composição corporal, mantendo a massa } \\
\text { magra e evitando o ganho excessivo de } \\
\text { massa gorda. A estreita ligação entre o } \\
\text { dentista e o nutricionista é fundamental. O } \\
\text { trabalho em equipe deve ser mantido e } \\
\text { incentivado para garantir o monitoramento } \\
\text { cuidadoso e a intervenção precoce do } \\
\text { tratamento, sempre que possível. }\end{array}$ \\
\hline
\end{tabular}




\begin{tabular}{|c|c|c|c|c|}
\hline $\begin{array}{l}\text { Hereditary } \\
\text { epidermolysisbul } \\
\text { losa in school } \\
\text { children and } \\
\text { adolescents. } \\
\text { Clinical picture } \\
\text { and } \\
\text { interdisciplinary } \\
\text { management }\end{array}$ & $e^{2016 .}$ & 2016 & PubMED & $\begin{array}{l}\text { A partir dos } 10 \text { anos de idade, crianças e } \\
\text { adolescentes com EBdistrófica generalizada } \\
\text { gravedevem receber rastreamentoregular de } \\
\text { tumor de pele. Pacientes com EB hereditária } \\
\text { requerem uma atenção regular e } \\
\text { interdisciplinar. Para evitar o déficit de } \\
\text { crescimento do paciente acometido, é } \\
\text { recomendável realizar a higiene dental de } \\
\text { forma adequada, além de aconselhamento } \\
\text { nutricional, para assim garantir uma } \\
\text { nutrição adequada. }\end{array}$ \\
\hline
\end{tabular}

Autoria própria (2021).

Além da escassez de publicações científicas sobre a temática, os resultados mostram que a abordagem sobre a atuação multidisciplinar no manejo da patologia ainda é citada de forma discreta nos estudos científicos, pois apesar desta atuação ser considerada importante para proporcionar aos indivíduos um tratamento ainda mais efetivo, são poucas as relações expostas nos estudos entre os profissionais de saúde que podem contribuir para o tratamento do indivíduo acometido e dos seus familiares.

Este achado corrobora com o estudo de BARROS, RASKIN \& PEREIRA (2004), ondefoi possível evidenciar que, apesar da alta complexidade que envolve a EB, a literatura ainda apresenta poucos estudos que envolvem essa temática, assim como também apresentam estudos com linguagem de difícil compreensão para a sociedade.

Para AMARAL, ANDRADE \& BARBOSA (2014), é recomendável realizar um acompanhamento cuidadoso da criança que apresente lesões, desde os primeiros dias de vida, assim objetivando chegar ao diagnóstico preciso da EB, para que possa ser iniciado o atendimento apropriado de forma mais precocemente possível. Além disso, ALVES et al. (2007) recomenda que em famílias que possuem indivíduos acometidos com a EB, o aconselhamento genético é muito importante, principalmente quando houver o desejo de gravidez, deixando claro o risco de se ter uma criança com a doença.

O estudo de AMARAL, ANDRADE \& BARBOSA (2014), evidencia que para garantirque o indivíduo com EB receba um cuidado adequado, é necessário atentar para vários fatores, tais como: banho, vestuário, alimentação, trato gastrointestinal, higiene oral, prevenção e cuidados com as bolhas, além dos aspectos psicológicos. 
O cuidado integral previne o surgimento de sequelas e proporciona maior qualidade de vida do indivíduo. O Estudo evidencia ainda que a abordagem multiprofissional se faz fundamental nesse processo.

Os profissionais envolvidos no tratamento dos indivíduos com EB devem propiciar a estes e aos seus familiares uma compreensão precisa da doença, bem como dos cuidados necessários no âmbito domiciliar, a fim de se prevenir o aparecimento de sequelas decorrentes das bolhas, proporcionando maior qualidade de vida para o indivíduo acometido e evitando possíveis complicações (AMARAL, ANDRADE \& BARBOSA, 2014).

Dentre os profissionais presentes na equipe multidisciplinar, pode-se destacar a atuação do fisioterapeuta, sendo este o profissional que direcionará as suas condutas terapêuticas para o tratamento do déficit motor gerado pelas limitações funcionais desencadeadas pelas bolhas, dessa forma tendo como objetivo prevenir maiores deformidades e limitações.

A fisioterapia é muito importante para o reforço das articulações, principalmente quando estas áreas apresentam cicatrizes. $\mathrm{Na}$ articulação temporomandibular, os benefícios dos exercícios fisioterápicos permitem reforço da musculatura, movimentos mandibulares corretos e desenvolvimento equilibrado (ALVES et al., 2007, pag.34).

A criação de um programa de exercícios fisioterapêuticos torna-se essencial, na medida em que contribui não somente para melhora das habilidades motoras, mas também proporcionando uma melhor qualidade de vida e de socialização dos pacientes. (SILVA et al., 2011).

O estudo de BEGA et al. (2015) evidencia que dentro da equipe multidisciplinar, os profissionais da Enfermagem possuem também um papel de grande importância no tratamentoda EB.

Os profissionais da área da enfermagem possuem papel importantíssimo em setratando de EB, uma vez que esses profissionais participam ativamente de todo o cuidado desses pacientes, seja no alívio e controle da dor, seja na observação de sinais de início de infecção e intervindo sobre estes, seja perfurando as bolhas quando necessário, realizando os curativos de forma adequada, aliviando as pressões sobre aslesões, cuidando das feridas, observando mudanças nas mesmas 
e amenizando o prurido, orientando e oferecendo apoio a esses pacientes e seus familiares, considerando o benefício da orientação adequada aos pais, do acesso a recursos adequados para a realização de curativos, alimentação adequada, prevenção de complicações clínicas e aporte multiprofissional para o regime domiciliar (BEGA et al. 2015, pag. 1).

\section{CONCLUSÃO}

Com a realização deste estudo, pode-se concluir que a EB é uma patologia que causa um grande impacto na vida dos indivíduos acometidos e dos seus familiares. Por se tratar de uma patologia crônica, hereditária e que não dispõe de cura, o tratamento desta requer uma intervenção multidisciplinar para garantir ao indivíduo acometido e os seus familiares um tratamento efetivo.

Pode ser evidenciado ainda que a literatura apresenta uma escassez de estudos científicos sobre esta temática, principalmente sobre a atuação multiprofissional nos cuidados destes pacientes e seus familiares. Diante disso, surge a necessidade de realização de mais estudos sobre a $E B$, especialmente enfatizando a atuação multidisciplinar no manejo dapatologia, para que assim estes estudos possam apoiar a prática dos profissionais de saúde, uma vez que estes munidos desses conhecimentos, poderão atuar na prevenção e promoção da saúde desses pacientes e seus familiares de forma mais ampla e efetiva. 


\section{REFERÊNCIAS BIBLIOGRÁFICAS}

ALVES, Patrícia Valéria Milanezi et al. Atendimento multidisciplinar do paciente ortodônticocom epidermólise bolhosa. Revista Dental Press de Ortodontia e Ortopedia Facial, v. 12, n.4, p. 30-35, 2007.

AMARAL, A. P.; ANDRADE, A. P. R.; BARBOSA, J. A. G. Epidermólise bolhosa: cuidados de enfermagem e orientações ao portador. Revista Tecer - Belo Horizonte - vol. 7 , $n^{\circ} 13,2014$.

BARBOSA, Gisele Cristine Teixeira et al. Epidermólise bolhosa distrófica e juncional: aspectos gastrointestinais. Pediatria (São Paulo), v. 27, n. 2, p. 87-94, 2005.

BARROS, Isabelle Rego; RASKIN, Salmo; PEREIRA-FERRARI, Lilian. Epidermólise Bolhosa Distrófica: Relato de um caso brasileiro. Estudos de Biologia, v. 26, n. 57, 2004.

BEGA, Aline Gabriela et al. Epidermólise bolhosa: revisão de literatura. Anais Eletrônico IX EPCC-Encontro Internacional de Produção Científica UniCesumar Nov, n. 9, p. 4-8, 2015.

BOEIRA, Vanessa Lys Simas Yamakawa. Epidermólise bolhosa hereditária: uma revisão de literatura. 2012.

CERVO, A. L.; BERVIAN, P. A. Metodologia científica. 5. ed. São Paulo: Prentice Hall, 2002.

DAS, B.; SAHOO, S. Dystrophic Epidermolysis Bullosa. Journal of Perinatology, 24(1), 41-47, 2004.

HUBBARD, L. et al. The challenges of meeting nutritional requirements in children and adultswith epidermolysis bullosa: proceedings of a multidisciplinary team study day. Clinical and Experimental Dermatology: Clinical dermatology, v. 36, n. 6, p. 579-584, 2011.

OLIVEIRA, Zilda Najjar Prado de et al. Imunomapeamento nas epidermólises bolhosas hereditárias. A. Bras. Dermatol. , Rio de Janeiro, v. 85, n. 6, pág. 856-861, dezembro de 2010.

OTT, H. et al. Hereditary epidermolysis bullosa in school children and adolescents. Clinical picture and interdisciplinary management. Der Hautarzt; Zeitschrift fur Dermatologie, Venerologie, und verwandte Gebiete, v. 67, n. 4, p. 279-286, 2016.

SILVA, D. M. et al. Proposta de um programa de exercícios fisioterapêuticos para crianças com epidermólise bolhosa. XII Salão de Iniciação Científica - PUCRS, 03 a 07 de outubro de 2011.

TOMASI, Neusi Garcia Segura; YAMAMOTO, Rita Miako. Metodologia da pesquisa em saúde: fundamentos essenciais. Curitiba: As autoras, 1999. 